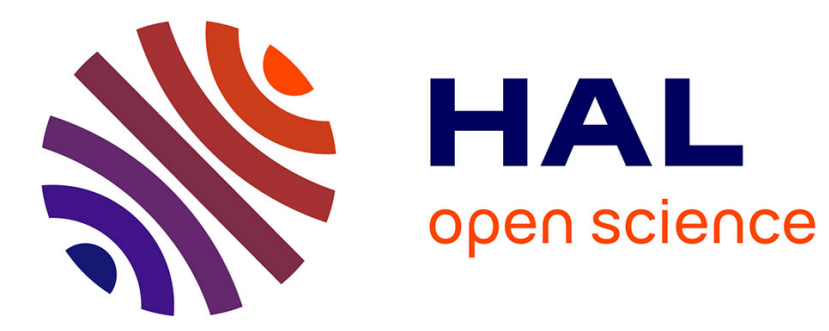

\title{
La prise en compte de la dimension linguistique dans les disciplines non linguistiques : du prescrit au réel
}

Julien Basso

\section{To cite this version:}

Julien Basso. La prise en compte de la dimension linguistique dans les disciplines non linguistiques: du prescrit au réel. Contextes et Didactiques, 2014. hal-01633228

\section{HAL Id: hal-01633228 \\ https://hal.science/hal-01633228}

Submitted on 12 Nov 2017

HAL is a multi-disciplinary open access archive for the deposit and dissemination of scientific research documents, whether they are published or not. The documents may come from teaching and research institutions in France or abroad, or from public or private research centers.
L'archive ouverte pluridisciplinaire HAL, est destinée au dépôt et à la diffusion de documents scientifiques de niveau recherche, publiés ou non, émanant des établissements d'enseignement et de recherche français ou étrangers, des laboratoires publics ou privés. 


\title{
La prise en compte de la dimension linguistique dans les disciplines non-linguistiques : du prescrit au réel
}

\author{
Julien BASSO \\ Université des Antilles et de la Guyane
}

\section{Résumé}

Le présent article propose une réflexion sur l'appropriation du français en contexte scolaire, et s'interroge plus spécifiquement sur la prise en compte de la dimension linguistique dans les disciplines non linguistiques (DNL) dans le contexte scolaire en Outre-Mer français. Elle présente une étude de cas menée dans un collège du sud Basse-Terre en Guadeloupe, qui vise à vérifier si, et dans quelle mesure, la dimension linguistique, pour ce qui relève de la compétence de production écrite (PE), est prise en compte et intégrée dans l'enseignement de trois DNL : Histoire, Géographie, et Sciences de la Vie et de la Terre, en classe de $6^{\text {ème }}$. L'analyse proposée utilise l'approche curriculaire comme méthodologie de travail : elle interroge la prise en compte de la dimension linguistique aux trois niveaux curriculaires, prescrit, planifié et réel, au regard des besoins des apprenants et au regard de la demande institutionnelle liée à l'implantation du socle commun des connaissances et des compétences. Cette étude, réalisée à partir de l'analyse des pratiques déclarées de trois enseignants, a pour but de vérifier si, et sous quelles formes, les enseignants de DNL planifient et intègrent dans leur enseignement la dimension linguistique, et quels types de compétences ils mobilisent, ou non, dans cette perspective. Les résultats de cette étude permettent de mettre en évidence les difficultés rencontrées par les enseignants pour une prise en compte effective de la dimension linguistique dans leurs spécialités respectives, mais également leur positionnement et leurs représentations sur ce point aux plans pédagogique et didactique. Ils permettent également de proposer des pistes de réflexion et des perspectives d'approfondissement, notamment sur la question de la formation des enseignants de DNL pour répondre aux besoins spécifiques des apprenants dans une situation de contacts de langues.

\section{Mots clés}

Approche curriculaire, compétences linguistiques, curriculum, discipline non linguistique, français langue de scolarisation, langues en contact, pedagogical contents knowledge.

\begin{abstract}
This article proposes a discussion about the French language appropriation in a school context. More specifically, it examines the linguistic dimension being taken into account in non linguistic disciplines (NLD), in the French overseas school context. It presents a case study led in a public school, in south Basse-Terre, Guadeloupe, which aims at verifying if and to what extent, the linguistic dimension, within the written competence, is taken into account and integrated in the teaching of 3 non linguistic disciplines : History, Geography, and Life and Earth Sciences, in Year 7. The methodology used in the analysis proposed is based on the curricular approach. It examines the extent of the linguistic dimension at various curricular levels : prescribed curriculum, planned curriculum and received curriculum, towards the need of the learners, and towards the institutional demand, which is linked to the implementation of the common basis of knowledge and skills. This study is based on the analysis of 3 teachers stated practices. It aims at verifying whether, and in what form, non linguistic disciplines teachers plan and integrate in their teaching the linguistic dimension. The results of this research highlight the difficulties encountered by the teachers to efficiently integrate the linguistic dimension in their own specialities, but also their positions and views on this matter at a pedagogical and didactic level. Furthermore the results propose areas for discussion and prospects deepening the discussion about the NLD teachers training, in order to meet the needs of learners in a case of languages in contact.
\end{abstract}

\section{Keywords}

Curricular approach, linguistic competences, curriculum, non linguistic disciplines, French as language of education, languages in contact, Pedagogical Contents Knowledge. 


\section{Introduction}

Le présent article aborde la question de l'appropriation du français comme langue de scolarisation en Outre-mer français. Elle présente une étude de cas menée dans un collège du sud Basse-Terre en Guadeloupe, dans laquelle nous nous interrogeons sur la prise en compte de la dimension linguistique dans les disciplines non linguistiques. Nous considérons du point de vue des apprentissages scolaires une situation de contacts de langues, dans laquelle se pose la question des choix didactiques et pédagogiques, qui pourraient être mis en œuvre dans une démarche d'adaptation et de contextualisation des enseignements au niveau de l'acquisition des compétences linguistiques.

La Guadeloupe est marquée par un contexte sociolinguistique caractérisé par le contact des langues créole et français. Cette situation pose la question de l'acquisition des compétences linguistiques et langagières, et par là même de la construction des savoirs disciplinaires des apprenants dans un système scolaire où le français est langue d'enseignement exclusive.

Si la maîtrise du français apparaît comme un objectif prioritaire sur le plan institutionnel au sein de l'Académie de Guadeloupe, le constat global, corrélé par les résultats académiques aux évaluations en français à l'entrée en $6^{\text {ème }}$, est celui d'un rendement modeste en termes d'acquisition des compétences en français dans le contexte scolaire. Le profil sociolinguistique des apprenants associé à d'autres éléments de types structurels et organisationnels - volumes horaires par champs disciplinaires, organisation des groupes classes, nombre et répartition des apprenants, taux d'encadrement - pourraient constituer des faisceaux d'explications. Néanmoins, nous tendons à considérer qu'un renouvellement des pratiques existantes et des orientations didactiques et pédagogiques adaptées, seraient susceptibles d'inverser la tendance et de favoriser le processus d'appropriation et d'acquisition par les apprenants des compétences scolaires attendues en français, et ce notamment dans le cadre des disciplines non linguistiques (désormais DNL).

C'est à cette problématique que nous nous sommes attachés dans le cadre de cette étude : nous nous interrogeons sur l'exploitation et les apports possibles des DNL, comme l'histoire, la géographie, ou les Sciences de la Vie et de la Terre (désormais SVT) dans le domaine de la maîtrise de la langue dans le contexte scolaire. Dans cette perspective, nous cherchons à étudier la place de la dimension linguistique à différents niveaux curriculaires, dans les DNL en classe de $6^{\text {ème }}$ : au niveau de la connaissance et de la prise en compte par les enseignants des recommandations et des programmes institutionnels d'une part, au niveau des actions planifiées par les enseignants d'autre part, et enfin au niveau de la mise en œuvre opérationnelle de ces actions en situation de classe. En d'autres termes, et plus précisément, nous cherchons à étudier les possibilités et les conditions qui permettraient d'intégrer la composante linguistique de manière effective et opérationnelle dans les enseignements non linguistiques, pour favoriser à la fois la construction des savoirs disciplinaires et l'acquisition des compétences linguistiques par le biais des DNL.

Notre démarche tente de répondre à une double problématique : le but de notre réflexion est d'abord et prioritairement axé sur une meilleure prise en compte du contexte d'enseignementapprentissage et des besoins des apprenants dans une situation de contacts de langues relevant des problématiques associées aux bi-plurilinguismes. Il tente parallèlement de proposer des pistes de réflexion pour une réponse adaptée à l'évolution de la demande institutionnelle que constitue l'implantation du Socle Commun des Connaissances et des Compétences dans le système scolaire français en Outre-Mer. Notre hypothèse repose sur l'idée qu'une approche 
fondée sur les principes d'une didactique d'intégration langue-DNL permettrait d'avoir une incidence sur les processus d'acquisition des compétences en français des apprenants dans le cadre scolaire.

Le travail que nous présentons se situe donc dans une démarche d'analyse et de recherche d'amélioration des stratégies et des pratiques pédagogiques en contexte scolaire. Dans le cadre de notre démarche, nous adoptons la didactique du curriculum comme approche de recherche. Nous cherchons à vérifier, au regard des documents institutionnels de référence, et au regard des besoins des apprenants, dans quelle mesure l'enseignement des DNL intègre de manière effective la dimension linguistique, et par là même à identifier les connaissances et les compétences mobilisées par les enseignants dans cette optique aux différents niveaux de la construction curriculaire : prescrit, planifié, et réel.

Après avoir précisé le contexte dans lequel notre projet de recherche a été mis en place et les différentes composantes qui ont fait émerger le besoin de mettre en œuvre cette démarche et cette perspective d'ingénierie pédagogique, nous présenterons les objectifs de notre étude, la problématique et les hypothèses qui ont guidé notre démarche, le cadre théorique sur lequel nous nous sommes appuyés, et la méthodologie que nous avons adoptée pour mener à bien notre travail d'analyse. Dans la dernière partie, nous présenterons les résultats de la recherche obtenus, et tenterons de proposer, à partir des données recueillies, des pistes de réflexion sur le choix de stratégies d'apprentissage adaptées au contexte d'enseignement ciblé, sur les conditions de leur mise en œuvre dans le cadre des DNL et leur impact potentiel sur le processus d'acquisition des apprenants en français dans le contexte scolaire guadeloupéen.

\section{Contexte de la recherche}

L'étude que nous présentons est à replacer dans un double contexte, à la fois institutionnel, lié à l'implantation du Socle Commun des Connaissances et des Compétences, et sociolinguistique, lié au contact des langues créole-français et aux répertoires langagiers des apprenants. $\mathrm{Ce}$ double contexte présente des enjeux en interaction qui amènent à s'interroger sur la place et le traitement accordés à la dimension linguistique dans le cadre des enseignements scolaires, et notamment dans le cadre des DNL.

\subsection{Le contexte institutionnel}

\subsubsection{L'implantation du socle commun : une demande institutionnelle en évolution}

Le Socle Commun des Connaissances et des Compétences a été introduit par le décret 2006$830 \mathrm{du} 11$ juillet 2006 dans le cadre de la loi d'orientation sur l'avenir de l'Ecole du 23 avril 2005. Après plusieurs années d'expérimentation, son implantation et sa mise en application ont été validées et généralisées à l'ensemble des établissements scolaires à partir de juin 2010. Les éléments du socle commun ont été intégrés aux programmes du collège depuis 2009, dans la continuité de ceux de l'école primaire publiés en 2008. Depuis 2011, la maîtrise et la validation des compétences décrites dans le socle sont nécessaires à l'issue de la classe de $3^{\text {ème }}$ pour obtenir le Diplôme National du Brevet (DNB).

Conçu comme un outil référentiel, le Socle Commun des Connaissances et des Compétences présente «ce que tout élève doit savoir et maîtriser à la fin de la scolarité obligatoire et constitue l'ensemble des connaissances, compétences, valeurs et attitudes nécessaires pour réussir sa scolarité, sa vie d'individu et de futur citoyen. » Il s'organise en sept domaines de 
compétences, et décrit pour chaque domaine les savoirs et connaissances essentiels à acquérir, ainsi que les capacités à développer et les attitudes nécessaires pour les mettre en œuvre.

Parmi les sept domaines de compétences définis par le socle commun, la compétence Maîtrise de la langue (cf. Tableau 1), qui constitue l'une des composantes de notre réflexion, est présentée comme une priorité absolue. Elle ne concerne pas seulement la discipline Français dans une conception qui se voudrait disciplinaire et cloisonnée, mais elle est au contraire envisagée de manière transversale et transdisciplinaire. Se pose ainsi la question de son intégration dans le cadre des DNL et des implications que celle-ci comporte sur les plans didactique et pédagogique en termes de définition des objectifs pédagogiques, de stratégies d'apprentissage et de mise en place des processus d'acquisition et d'évaluation des compétences visées.

\begin{tabular}{|c|c|}
\hline DOMAINES & ITEMS ASSOCIES \\
\hline \multirow{5}{*}{ LIRE } & Adapter son mode de lecture à la nature du texte proposé et à l'objectif poursuivi \\
\hline & $\begin{array}{l}\text { Repérer des informations dans un texte à partir de ses éléments explicites et des éléments } \\
\text { implicites nécessaires }\end{array}$ \\
\hline & $\begin{array}{l}\text { Utiliser ses capacités de raisonnement, ses connaissances sur la langue, savoir faire appel à des } \\
\text { outils appropriés pour lire }\end{array}$ \\
\hline & Dégager, par écrit ou oralement, l'essentiel d'un texte lu \\
\hline & Manifester par des moyens divers sa compréhension de textes variés \\
\hline \multirow{4}{*}{ ECRIRE } & Reproduire un document sans erreur avec une présentation adaptée \\
\hline & $\begin{array}{l}\text { Ecrire lisiblement un texte, spontanément ou sous la dictée, en respectant l'orthographe et la } \\
\text { grammaire }\end{array}$ \\
\hline & $\begin{array}{l}\text { Rédiger un texte bref, cohérent et ponctué, en réponse à une question, ou à partir de consignes } \\
\text { données }\end{array}$ \\
\hline & $\begin{array}{l}\text { Utiliser ses capacités de raisonnement, ses connaissances sur la langue, savoir faire appel à des } \\
\text { outils variés pour améliorer son texte }\end{array}$ \\
\hline \multirow{4}{*}{ DIRE } & Formuler clairement un propos simple \\
\hline & Développer de façon suivie un propos en public sur un sujet déterminé \\
\hline & Adapter sa prise de parole à la situation de communication \\
\hline & Participer à un débat, un échange verbal \\
\hline
\end{tabular}

Tableau 1 : Socle Commun des Connaissances et des Compétences Compétence 1 : La maîtrise de la langue (janvier 2011) ${ }^{1}$

\subsubsection{Implications sur les plans pédagogique et didactique}

L'introduction du Socle Commun des Connaissances et des Compétences (SCCC) au collège marque en effet une évolution importante dans la politique éducative menée dans le cadre de 1'Éducation Nationale en France. Les directives relatives à sa mise en œuvre dans les enseignements scolaires au collège mettent en avant des perspectives nouvelles axées sur des principes clés qui constituent les repères des exigences institutionnelles en matière de politique éducative en France, mais également au niveau européen et international. Quatre mots clés

\footnotetext{
${ }^{1}$ Direction Générale de l'Enseignement Scolaire (2011, janvier). Grille de référence des compétences et des connaissances, Palier 3. Paris : Ministère de l'Education Nationale.
} 
caractérisent les principes et les nouvelles exigences institutionnelles qui sous-tendent l'implantation du Socle Commun ${ }^{2}$ :

Transversalité : les compétences recouvrent plusieurs disciplines, elles s'exercent dans des situations variées.

Contextualisation / Décontextualisation: la compétence doit être maîtrisée et évaluée à travers des situations concrètes, les plus proches possible de celles rencontrées dans la vie réelle.

Complexité: les tâches, les situations de mise en ouvre des compétences sont par essence complexes, requérant la mobilisation de savoirs, savoir faire, capacités, attitudes variées.

Intégration: les compétences intègrent diverses disciplines, diverses facettes (capacités, attitudes, connaissances).

Le Socle Commun des Connaissances et des Compétences implique ainsi une orientation nouvelle en termes d'approches didactiques et méthodologiques, de pratiques pédagogiques, de modalités et de stratégies d'évaluation, et conduit par conséquent à s'interroger sur la conception des formes d'enseignement mises en œuvre dans le contexte scolaire dans sa dimension curriculaire. C'est à cette question que s'attache notre travail pour ce qui relève de l'acquisition des compétences linguistiques dans le cadre des DNL.

\subsection{Un contexte sociolinguistique et des besoins spécifiques}

Outre la nécessité de répondre à une demande institutionnelle en évolution, notre travail est également lié à la recherche d'une meilleure prise en compte du profil des apprenants et du contexte sociolinguistique dans lequel ils évoluent. Le contexte sociolinguistique à l'œuvre en Guadeloupe est caractérisé par une situation de langues en contact où français et créole coexistent et se chevauchent dans les différentes situations de communication au quotidien. Cette situation de contacts de langues et les problématiques qu'elle génère sont au cœur du système scolaire amènent à s'interroger sur les orientations possibles en termes de stratégies d'apprentissage et d'approches didactiques dans une démarche de contextualisation des apprentissages et de prise en compte des besoins des apprenants (Sainton-Facthum, 2008a, 2008 b Prudent, Tupin et Wharton, 2005).

Or le système scolaire, qui sur le plan institutionnel peut être caractérisé comme une situation d'apprentissage par immersion (Gajo, 2001), le français étant la langue unique de transmission des savoirs et des connaissances scolaires, ne reconnaît pas formellement les spécificités du contexte d'enseignement-apprentissage à l'œuvre en Guadeloupe. Il n'existe par conséquent que très peu d'outils à disposition des enseignants pour tenter de répondre didactiquement et pédagogiquement aux besoins dont il relève. Ce contexte spécifique nous amène donc à nous interroger du point de vue des apprentissages scolaires sur la question de l'appropriation du français comme langue de scolarisation dans un contexte relevant des situations de bi/plurilinguisme. Par Français Langue de Scolarisation (FLSco), on entend une variété normée du français définie comme « langue enseignée et langue d'enseignement, mais aussi et surtout comme langue de communication spécifique au milieu scolaire » (Vigner, 1989), à l'oral et à l'écrit. La mise en place d'une démarche relevant du FLSco implique par conséquent le

\footnotetext{
${ }^{2}$ Inspection Générale de l'Education Nationale, Les livrets de compétences, nouveaux outils pour l'évaluation des acquis (Rapport $\mathrm{N}^{\circ}$ 2007-048, juin 2007). Paris : Ministère de l’Education Nationale.
} 
développement de compétences scolaires spécifiques qui englobent des compétences linguistiques, mais également des compétences fonctionnelles et stratégiques nécessaires à la réalisation des tâches scolaires dans les différents champs disciplinaires.

Les enseignants des disciplines linguistiques, mais également des disciplines non linguistiques se trouvent ainsi confrontés à la gestion et au traitement des difficultés liées au contexte sociolinguistique en présence. De nombreux travaux montrent, analysent et décrivent l'impact potentiel du contexte sociolinguistique sur l'acquisition des compétences langagières attendues dans le cadre scolaire en Guadeloupe, et plus largement aux Antilles françaises, et tentent d'apporter des réponses adaptées ou des pistes de réflexion pour une contextualisation didactique et pédagogique (Cambrone, 2004 ; Prudent, Tupin et Wharton, 2005 ; Blanchet, Moore et Asselah-Rahal, 2008; Sainton-Facthum, Gaydu et Chery, 2010). Parmi eux, les travaux menés par Anciaux $(2005,2007,2008,2010)$ qui étudie les phénomènes d'alternances codiques dans les situations d'apprentissage en contexte scolaire, placent l'utilisation de l'alternance codique au cœur des pratiques et l'identifient comme une stratégie didactique possible pour favoriser à la fois l'acquisition des compétences linguistiques mais également des savoirs et des connaissances disciplinaires dans le cas des disciplines non linguistiques. Cette approche, qui consiste dans le croisement de la didactique des langues et des didactiques disciplinaires, et qui propose une «construction raisonnée de l'alternance des langues en fonction des tâches spécifiques à accomplir, de manière à favoriser les processus d'enseignement/apprentissage en contexte bi/plurilingue en offrant une plus grande variété de moyens de transmission et de construction de connaissances " (Anciaux, 2010) relève de la didactique intégrée des langues (Candelier, 2008 ; Chiss, 2001 ; Cavalli, 2005 ; Gajo, 2001, 2006 ; Causa, 2007, 2009 ; Brohy, 2008 ; Wokusch, 2005, 2008).

Dans le cadre de notre projet, si nous considérons en effet qu'une approche relevant de la didactique intégrée des langues serait une réponse possible (mais non exclusive) adaptée aux problématiques générées par le contexte sociolinguistique et le profil des apprenants auxquels nous sommes confrontés en Guadeloupe, nous proposons d'aborder la question sous une approche curriculaire. Cette dernière, considérant qu'une (ré)orientation des pratiques pédagogiques mises en place dans le cadre des DNL et intégrant le développement des compétences linguistiques et langagières à la construction des savoirs disciplinaires, permettrait d'améliorer le processus d'acquisition des apprenants en français dans le contexte scolaire.

C'est dans ce double contexte, déterminé par des enjeux à la fois institutionnels et sociolinguistiques, que se situe notre objet d'étude. Les problématiques à l'œuvre sont complexes et relèvent de différentes composantes qui peuvent être abordées sous des angles multiples. La démarche que nous proposons consiste essentiellement à tenter de dresser un état des lieux de la situation existante dans un établissement cible, où se croisent et interagissent les problématiques liées à la fois à l'évolution de la demande et des exigences institutionnelles, aux besoins de formation spécifiques liés à l'environnement scolaire, et au profil d'apprenants relevant de situations de bilinguisme et de diglossie, dans une réflexion axée sur le domaine spécifique de la maîtrise de la langue en contexte scolaire dans les DNL. La complexité d'un tel contexte rend nécessaire une délimitation précise et rigoureuse des objectifs que nous nous sommes fixés, de la problématique qui sous-tend notre travail et des hypothèses qui orienteront notre démarche. 


\section{Objectifs et problématique}

Notre recherche interroge la prise en compte de la dimension linguistique dans les DNL pour ce qui relève de la compétence de Production Écrite (désormais PE), au niveau des pratiques pédagogiques et des formes d'enseignement planifiées, puis mises en œuvre de façon effective par les enseignants de DNL. Nous ne cherchons pas dans le cadre de notre travail à recenser des pratiques pédagogiques identifiées pour en dresser une typologie, mais à étudier les articulations et les interactions éventuelles entre le prescrit et les pratiques réelles déclarées des enseignants. Cette approche amène à s'interroger sur les implications de la prise en compte et de l'intégration de la dimension linguistique dans les DNL au niveau didactique et pédagogique, mais également au niveau du profil des enseignants en termes de savoirs et de savoir-faire. L'intégration d'une composante Langue dans les DNL peut être en effet considérée ou définie comme un élargissement de spécialité, ce qui oriente notre réflexion vers la question de la délimitation de cet élargissement de spécialité, et sur le degré d'expertise et de compétences nécessaires à l'enseignant pour mettre en œuvre un enseignement de ce type (Coquidé, 2011) et contribuer dans le cadre de sa spécialité non linguistique à l'acquisition et au développement des compétences linguistiques, à l'écrit notamment, des apprenants : c'est à ce niveau que se situe la problématique de notre travail. Il s'agit pour nous de tenter d'identifier à partir d'une situation existante les compétences et les connaissances mobilisées et/ou à mobiliser par les enseignants pour mettre en place une pratique intégrant la dimension linguistique, tenant compte d'une part du prescrit institutionnel, mais également des besoins des apprenants sur le plan de la maîtrise de la langue.

Notre travail est une étude exploratoire. Nous ne cherchons pas à vérifier une hypothèse préétablie, mais à mettre en évidence des indicateurs en lien avec notre problématique, et qui permettront de caractériser une situation existante dans un contexte d'enseignementapprentissage donné. À ce stade de notre travail, nous pouvons tout au plus envisager différents cas de figures, que l'analyse des données que nous avons recueillies nous permettra, ou non, de vérifier :

- sur la connaissance, la maîtrise et la prise en compte ou non du prescrit par les enseignants de DNL pour ce qui relève de la composante linguistique au niveau PE ;

- sur la planification ou non par les enseignants de DNL de pratiques pédagogiques en lien avec le développement de la composante linguistique au niveau PE;

- sur l'intégration ou non par les enseignants de DNL dans leurs pratiques pédagogiques effectives de la composante linguistique au niveau PE ;

- sur les corrélations qui existent entre les contenus du prescrit et l'intégration par les enseignants de DNL dans leurs pratiques effectives de la composante linguistique PE ;

- sur les conceptions et représentations de la dimension linguistique et de son intégration dans leurs pratiques chez enseignants de DNL.

Nous situons l'ensemble de ces questionnements, qui constituent le cœur de notre problématique, dans un double cadre théorique, la didactique du curriculum d'une part, et les PCK (Pedagogical Contents Knowledge) d'autre part, dont nous présentons ci-dessous les principes sous-jacents en lien avec les données que nous cherchons à analyser. 


\section{Cadre théorique}

\subsection{La didactique du curriculum}

Nous situons notre travail dans le cadre de la didactique du curriculum. Nous cherchons à vérifier dans quelle mesure la dimension linguistique et l'acquisition des compétences en français des apprenants est prise en compte et intégrée à l'enseignement des DNL. Notre travail interroge à la fois: les pratiques réelles déclarées des enseignants en termes de programmation/planification et en termes de mise en œuvre opérationnelle des enseignements, ainsi que le processus de transfert et de transposition de l'un à l'autre, qui intègre les savoirs et savoir-faire mobilisés par l'enseignant.

La nature des données que nous cherchons à identifier, nous conduit à mener notre analyse sous une approche curriculaire. Nous nous appuyons dans le cadre de notre étude sur la distinction des quatre niveaux curriculaires définis par Martinand (2003) :

- le curriculum prescrit, défini par le contenu des programmes disciplinaires et du socle commun des connaissances et des compétences ;

- le curriculum potentiel, qui désigne les choix et les prises de décisions effectués par les enseignants pour la mise en œuvre du projet pédagogique et éducatif dans les différents champs disciplinaires ;

- le curriculum produit, qui correspond à la traduction du curriculum potentiel en une programmation/progression de séquences structurées, avec des objectifs d'apprentissage et des activités pédagogiques définis et planifiés. Le curriculum produit est fixé sur des documents type programmations annuelles, descriptifs de séquences pédagogiques, cahiers de bords, fiches d'activités ;

- le curriculum effectif, ou réel, qui correspond à la prise en charge par l'enseignant du curriculum produit, transposé en situation de classe.

Toutefois, nous n'intégrons pas dans notre recherche le $2^{\text {ème }}$ niveau curriculaire ou curriculum potentiel. En effet, notre objectif ne consiste pas dans l'exploration des possibilités de choix offertes aux enseignants pour intégrer la dimension linguistique dans le cadre des DNL. Il vise à interroger une situation existante en focalisant notre questionnement sur l'action de planification, de conception et de mise en œuvre par les enseignants de DNL de pratiques relevant de l'apprentissage ou de l'appropriation de la langue à l'écrit dans le cadre de leur enseignement, au regard des contenus programmatiques et des attentes institutionnelles (curriculum prescrit) d'une part, mais également au regard des besoins spécifiques des apprenants dans un contexte d'enseignement-apprentissage caractérisé par le contact des langues créole et français.

Ce questionnement implique par conséquent d'identifier les connaissances et les compétences mobilisées et/ou à mobiliser par les enseignants pour intégrer dans l'enseignement des DNL la dimension linguistique et mettre en œuvre des pratiques favorisant l'acquisition par les apprenants des compétences PE. Pour mener à bien cette analyse, et compte tenu des données que nous cherchons à mettre en évidence, nous nous sommes appuyés sur les PCK de Shulman (1986), dont nous présentons ci-dessous le cadre théorique et méthodologique. 


\subsection{Les PCK (Pedagogical Contents Knowledge)}

Les PCK (Pedagogical Contents Knowlegde) ont été initiées par Shulman (1986) : ce cadre d'analyse nous permet de mettre en évidence les connaissances enseignantes mobilisées dans les DNL pour ce qui relève de la prise en compte de la dimension linguistique. Concernant les connaissances enseignantes ou PCK potentiellement mobilisées, Shulman (1986) définit les trois catégories suivantes :

- 1) La connaissance disciplinaire (SMK: Subject Matter Knowledge) est issue de la formation universitaire des enseignants. Ce sont les connaissances académiques de la discipline à enseigner.

- 2) La connaissance pédagogique (PCK : Pedagogical Knowledge) correspond à la transposition des connaissances scientifiques en des connaissances accessibles aux élèves via des illustrations, des démonstrations, la prise en compte des difficultés rencontrées par les élèves, etc. Il s'agit des pratiques et des stratégies pédagogiques planifiées, puis mises en œuvre de manière effective dans la classe, caractérisées par la définition des objectifs d'apprentissage, le choix des contenus et des types d'activités proposées, des supports pédagogiques, des démarches de guidage, ou encore des stratégies et des modalités d'évaluation.

- 3) La connaissance curriculaire (CK: Curricula Knowledge) désigne la connaissance des programmes scolaires et du matériel pédagogique disponible pour mettre en œuvre les programmes.

L'objectif de notre étude est double. Il consiste, d'une part, à identifier et à caractériser les éléments liés à la dimension linguistique relevant de la compétence $\mathrm{PE}$, dans les pratiques des enseignants, dans le processus de transposition du prescrit au réel. D'autre part, il vise à identifier et à caractériser les PCK mobilisées par les enseignants de DNL, et à étudier les articulations éventuelles entre les CK (connaissances curriculaires), les PK (connaissances pédagogiques) et les SMK (connaissances disciplinaires) pour ce qui concerne la prise en compte et l'intégration de la dimension linguistique dans les DNL aux différents niveaux curriculaires, prescrit, produit, et réel.

Il s'agit à partir de cette analyse de voir si, et dans quelle mesure, le prescrit, défini par les programmes officiels et par le socle commun des connaissances et des compétences, est connu, planifié (curriculum produit) puis transposé en pratiques réelles par les enseignants dans leurs spécialités respectives (curriculum réel). Les données recueillies doivent également nous permettre de vérifier s'il existe des articulations entre CK, SMK et PK des enseignants de DNL pour ce qui relève de l'apprentissage de la langue, et plus spécifiquement de la compétence PE. Et si oui, de quels types d'articulations s'agit-il ?

\section{Méthodologie}

Pour répondre à notre problématique, nous nous appuyons sur une étude de cas menée dans un collège de 280 élèves du sud Basse-Terre, situé en zone périurbaine, auprès d'une équipe de trois enseignants de DNL, dont deux enseignants d'histoire-géographie (E1, E2) et un enseignant de SVT (E3). Cette étude de cas repose sur l'analyse d'enregistrements audio d'entretiens réalisés individuellement avec chaque enseignant. Nous présentons ci-après la démarche que nous adoptons concernant le recueil, le traitement et l'analyse des données extraites des supports sélectionnés. 


\subsection{Sélection et recueil des données}

Le recueil s'appuie sur les enregistrements audio d'entretiens d'explicitation individuels de type semi-directif d'une durée totale d'environ deux heures, menés avec deux enseignants d'histoire-géographie (E1, E2) et un enseignant de SVT (E3). Ces entretiens ont pour but de recueillir les données relatives aux pratiques réelles déclarées des enseignants pour ce qui relève de la dimension linguistique : ils doivent nous permettre d'une part d'analyser sous l'approche curriculaire les formes d'enseignement mises œuvre par les enseignants de DNL, et d'autre part d'extraire des indicateurs relatifs aux PCK mobilisées aux différents niveaux curriculaires, prescrit, planifié et réel. Ils doivent également permettre de mettre en évidence le ressenti, le positionnement et les postures des enseignants face à la prise en compte de la dimension linguistique dans leur discipline respective, au regard de l'évolution de la demande institutionnelle liée à l'implantation du socle commun des connaissances et des compétences, et au regard des besoins des apprenants liés au contexte sociolinguistique.

\subsection{Analyse du corpus : grille de recueil et d'analyse des données}

L'analyse des entretiens réalisés avec les enseignants a pour objectif de mettre en évidence et de caractériser les PCK mobilisées par les enseignants dans les différentes phases de la construction curriculaire. Comme pour l'analyse du curriculum prescrit, nous adoptons une démarche d'analyse de contenu (Bardin, 1996 ; Sabourin, 2003). Cette analyse a été appliquée sur les discours des sujets interrogés, collectés lors des entretiens semi-directifs menés au cours de notre enquête. Nous cherchons par cette analyse à mettre en évidence des indicateurs qui permettent :

- 1) de vérifier si, et dans quelle mesure, la dimension linguistique relevant de la compétence PE est prise en compte et intégrée à l'enseignement des DNL dans le contexte d'enseignement-apprentissage que nous ciblons ;

- 2) de caractériser les types de connaissances et de compétences (PCK) mobilisées par les enseignants dans la prise en compte, la planification et la mise en œuvre effective des objectifs d'apprentissages et des pratiques pédagogiques en lien avec la compétence PE.

La trame de l'entretien a été élaborée et organisée en fonction des objectifs poursuivis, des thématiques que nous cherchons à explorer, et des données que nous cherchons à extraire. Elle est composée de plusieurs groupes de questions qui reflètent les principaux axes de notre recherche : il s'agit d'identifier les PCK mobilisées par les enseignants de DNL pour la prise en compte de la dimension linguistique dans leur spécialité respective, aux différents niveaux de la construction curriculaire : curriculum prescrit, curriculum produit, et curriculum réel.

Les données que nous cherchons à extraire à partir des entretiens nous ont amené à définir des indicateurs adaptés au contexte et aux besoins de notre recherche. Ce travail de clarification et de contextualisation a abouti à l'élaboration d'une grille d'analyse des PCK mobilisées par les enseignants de DNL pour ce qui relève de la prise en compte et de l'intégration de la compétence PE dans leur spécialité respective (cf. Tableau 2). 


\begin{tabular}{|c|c|c|c|c|c|c|}
\hline \multirow{2}{*}{$\begin{array}{c}\text { Niveaux } \\
\text { curriculaires }\end{array}$} & \multirow{2}{*}{ PCK mobilisées } & \multirow{2}{*}{ Caractéristiques } & \multirow{2}{*}{ Exemples d'indicateurs } & \multicolumn{3}{|c|}{ Etiquetage } \\
\hline & & & & E1 & E2 & E3 \\
\hline Prescrit & $\begin{array}{c}\text { CK } \\
\text { (Curriculum } \\
\text { Knowledge) }\end{array}$ & $\begin{array}{l}\text { Connaissances et prise } \\
\text { en compte des } \\
\text { documents } \\
\text { institutionnels } \\
\text { (programmes } \\
\text { disciplinaires et } \\
\text { contenus du SCCC) }\end{array}$ & $\begin{array}{l}\text { Connaissance des éléments du SCCC } \\
\text { en lien avec la } \\
\text { maîtrise/l'apprentissage de la langue } \\
\text { (compétence PE) } \\
\text { Connaissances et prise en compte des } \\
\text { objectifs d'apprentissage définis dans } \\
\text { les programmes officiels en lien avec } \\
\text { la maîtrise/l'apprentissage de la } \\
\text { langue (compétence PE) }\end{array}$ & $\begin{array}{l}\text { Q1.24 à } \\
\text { Q1.31 } \\
\text { Q1.42 } \\
\text { Q1.43 }\end{array}$ & $\begin{array}{l}\text { Q2.27 } \\
\text { Q2.28 }\end{array}$ & $\begin{array}{l}\text { Q3.9 à } \\
\text { Q3.15 } \\
\text { Q3.33 }\end{array}$ \\
\hline \multirow{3}{*}{$\begin{array}{l}\text { Produit } \\
\text { (planifié) }\end{array}$} & $\begin{array}{c}\text { SMK (Subject } \\
\text { Matter } \\
\text { Knowledge) } \\
\text { Langue } \\
\end{array}$ & $\begin{array}{l}\text { Connaissances liées au } \\
\text { domaine de } \\
\text { l'apprentissage de la } \\
\text { langue }\end{array}$ & $\begin{array}{l}\text { Connaissance des concepts, des } \\
\text { éléments méthodologiques, des } \\
\text { contenus à enseigner, des objectifs } \\
\text { d'apprentissage à définir }\end{array}$ & \multirow{3}{*}{$\begin{array}{l}\text { Q1.13 } \\
\text { Q1.14 } \\
\text { Q1.32 }\end{array}$} & \multirow{3}{*}{$\begin{array}{l}\text { Q2.9 } \\
\text { Q2.21 à } \\
\text { Q2.26 }\end{array}$} & \multirow{3}{*}{$\begin{array}{l}\text { Q3.37 } \\
\text { Q3.38 }\end{array}$} \\
\hline & $\begin{array}{l}\text { PK (Pedagogical } \\
\text { Knowledge) } \\
\text { Langue }\end{array}$ & $\begin{array}{l}\text { Connaissances } \\
\text { pédagogiques liées au } \\
\text { domaine de } \\
\text { l'apprentissage de la } \\
\text { langue : savoirs et } \\
\text { savoir-faire } \\
\text { nécessaires à la mise } \\
\text { en œuvre pédagogique }\end{array}$ & $\begin{array}{l}\text { Types d'activités planifiées, types de } \\
\text { tâches à réaliser, supports et outils } \\
\text { pédagogiques utilisés, types et } \\
\text { modalités d'évaluation (types de } \\
\text { productions/performances évaluées, } \\
\text { résultats attendus, critères évalués) }\end{array}$ & & & \\
\hline & $\begin{array}{c}\text { PCK } \\
\text { Collaboratif } \\
\text { (Collaborative } \\
\text { Knowledge) }\end{array}$ & $\begin{array}{l}\text { Compétences } \\
\text { mobilisées dans une } \\
\text { démarche } \\
\text { collaborative }\end{array}$ & $\begin{array}{l}\text { Prises de décisions communes, } \\
\text { concertation, échanges, démarches } \\
\text { d'harmonisation, dans le cadre de } \\
\text { projets communs de type } \\
\text { interdisciplinaire par exemple }\end{array}$ & & & \\
\hline \multirow[b]{2}{*}{$\begin{array}{c}\text { Réel } \\
\text { (effectif) }\end{array}$} & $\begin{array}{l}\text { SMK (Subject } \\
\text { Matter } \\
\text { Knowledge) } \\
\text { Langue }\end{array}$ & $\begin{array}{l}\text { Connaissances liées au } \\
\text { domaine de } \\
\text { l'apprentissage de la } \\
\text { langue }\end{array}$ & $\begin{array}{l}\text { Connaissance des concepts, des } \\
\text { contenus à enseigner, des éléments } \\
\text { métalinguistiques par exemple }\end{array}$ & $\begin{array}{l}\text { Q1.1 à } \\
\text { Q1.12 } \\
\text { Q1.18 à } \\
\text { Q1.20 }\end{array}$ & $\begin{array}{l}\text { Q2.1 à } \\
\text { Q2.8 } \\
\text { Q2.11 à } \\
\text { Q2.16 }\end{array}$ & \\
\hline & $\begin{array}{l}\text { PK (Pedagogical } \\
\text { Knowledge) } \\
\text { Langue }\end{array}$ & $\begin{array}{l}\text { Connaissances/compét } \\
\text { ences liées à la mise } \\
\text { en œuvre pédagogique }\end{array}$ & $\begin{array}{l}\text { Choix/intentions pédagogiques liés à } \\
\text { l'organisation de la classe en } \\
\text { situation, aux démarches adoptées } \\
\text { (degré de guidage, degré d'autonomie, } \\
\text { auto-régulation, démarche } \\
\text { collaborative, auto-correction, co- } \\
\text { correction, etc.), aux supports } \\
\text { pédagogiques utilisés (fiches } \\
\text { méthode, guides d'activités, outils } \\
\text { d'auto-évaluation) }\end{array}$ & $\begin{array}{l}\text { Q1.20 } \\
\text { Q1.21 } \\
\text { Q1.23 } \\
\text { Q1.33 à } \\
\text { Q1.38 }\end{array}$ & $\begin{array}{l}\text { Q2.11 à } \\
\text { Q2.16 } \\
\text { Q2.29 } \\
\text { Q2.30 }\end{array}$ & $\begin{array}{l}\text { Q3.4 à } \\
\text { Q3.8 } \\
\text { Q3.18 à } \\
\text { Q3.28 }\end{array}$ \\
\hline \multicolumn{2}{|c|}{ PCK Expertise } & $\begin{array}{l}\text { Capacités d'analyse et } \\
\text { de réflexion pour } \\
\text { identifier les } \\
\text { difficultés, les besoins } \\
\text { liés à la prise en } \\
\text { compte de la langue et } \\
\text { au développement des } \\
\text { compétence PE dans } \\
\text { les DNL }\end{array}$ & $\begin{array}{l}\text { Identification des besoins des } \\
\text { apprenants, des difficultés effectives } \\
\text { ou potentielles, des besoins de } \\
\text { formation des enseignants liés à la } \\
\text { prise en compte et à l'intégration de la } \\
\text { dimension linguistique dans les DNL, } \\
\text { éléments de réflexion sur les axes } \\
\text { d'amélioration possibles par rapport à } \\
\text { la situation existante }\end{array}$ & $\begin{array}{l}\text { Q1.15 à } \\
\text { Q1.17 } \\
\text { Q1.22 } \\
\text { Q1.39 à } \\
\text { Q1.41 } \\
\text { Q1.44 } \\
\text { Q1.45 }\end{array}$ & $\begin{array}{l}\text { Q2.10 } \\
\text { Q2.17 ̀̀ } \\
\text { Q2.20 }\end{array}$ & $\begin{array}{c}\text { Q3.2 } \\
\text { Q3.3 } \\
\text { Q3.16 } \\
\text { Q3.17 } \\
\text { Q3.29 à } \\
\text { Q3.32 } \\
\text { Q3.34 à } \\
\text { Q3.36 }\end{array}$ \\
\hline
\end{tabular}

Tableau 2 : grille d'analyse des compétences et connaissances (PCK) mobilisées par les enseignants de DNL en fonction des différentes phases de la construction curriculaire

Nous avons ainsi identifié 5 types de PCK répartis dans les différentes phases de la construction curriculaire :

- les Curriculum Knowledge (CK) au niveau du curriculum prescrit ;

- les Subject Matter Knowledge (SMK) et Pedagogical Knowledge (PK) au niveau du curriculum produit, auquel il nous a semblé pertinent d'intégrer des PCK 
« collaboratives » (Collaborative Knowledge), qui englobent les compétences mobilisées par les enseignants pour collaborer et élaborer un projet commun ;

- les SMK et PK au niveau du curriculum réel ;

- enfin, nous avons ajouté à cet ensemble un dernier type de PCK, que nous avons situé hors des trois niveaux curriculaires pris en compte, et qui relèvent de la capacité des enseignants de DNL à analyser la prise en compte de la dimension linguistique dans leur spécialité respective, à identifier des difficultés et des besoins éventuels au niveau apprenants et au niveau enseignants concernant la compétence PE : les PCK Expertise.

L'analyse des entretiens a été réalisée à partir de cette typologie. Les questions posées au cours de l'entretien, et les réponses apportées par les enseignants interviewés ont été étiquetées sous la forme Q1.X (question $\mathrm{N}^{\circ} \mathrm{X}$ posée à l'enseignant 1) et E1.X. (réponse $\mathrm{N}^{\circ} \mathrm{X}$ apportée par l'enseignant 1). Cet étiquetage a pour but à la fois de faciliter le repérage et le classement des éléments identifiés en fonction des indicateurs définis, et de vérifier la pertinence et l'adéquation des questions posées avec les objectifs poursuivis.

Enfin, nous précisons qu'au cours des entretiens, la nature et l'ordre des questions ont pu varier d'un enseignant à l'autre, en fonction du contenu des réponses apportées. Toutefois, nous avons choisi comme point de départ commun des entretiens d'inviter les interviewés à évoquer et à décrire une ou des séances pédagogiques réalisées en situation réelle en classe de $6^{\text {ème }}$.

\section{Résultats : analyse des pratiques réelles déclarées des enseignants et PCK mobilisées}

L'analyse des entretiens réalisés avait pour objectif de mettre en évidence des indicateurs permettant d'identifier et de caractériser les pratiques planifiées et réelles déclarées des enseignants et les PCK mobilisées pour la prise en compte et l'intégration des compétences PE dans l'enseignement des DNL. Cette analyse constitue le $2^{\text {ème }}$ volet de notre travail, après l'exploration des documents institutionnels de référence (curriculum prescrit).

Les résultats de cette analyse, s'ils constituent une base de données sans doute non exhaustive, nous permettent néanmoins de mettre en évidence des indicateurs qui nous renseignent sur les pratiques réelles déclarées des enseignants interrogés, sur les PCK mobilisées, mais également sur leurs difficultés dans la prise en compte et le traitement de la dimension linguistique, sur les stratégies qu'ils adoptent pour tenter d'y remédier, ainsi que sur leurs représentations de ce que doit être, ou pourrait être, l'intégration d'une composante linguistique dans l'enseignement de leur spécialité respective, compte tenu du contexte d'enseignement-apprentissage ciblé et du profil des apprenants sur le plan sociolinguistique.

Nous présentons ci-dessous les PCK identifiées au cours de l'analyse des entretiens des trois enseignants interviewés en fonction des différentes phases de la construction curriculaire : prescrit, produit et réel.

\subsection{Au niveau du curriculum prescrit : les CK (Curriculum Knowledge)}

Pour ce qui relève de la dimension linguistique, et plus spécifiquement des compétences PE pour le niveau $6^{\text {ème }}$, les indicateurs relevés au cours de l'analyse montrent que les trois enseignants interviewés s'appuient à la fois sur les contenus programmatiques et sur les contenus du SCCC pour définir des objectifs pédagogiques et des critères à évaluer. On note par ailleurs, concernant le SCCC, une tendance globale à associer la dimension linguistique au 
seul domaine de compétence C1/Maîtrise de la langue (extrait 1), sans prise en compte des autres domaines de compétences relevant potentiellement de la dimension linguistique, ce qui peut révéler une méconnaissance au niveau des SMK et des PK Langue. Ce point sera évoqué avec les résultats de l'analyse des PCK mobilisées au niveau du curriculum réel (cf. 5.3).

\section{Extrait 1 (entretien E2) :}

E2.27 Je vais sur le socle commun, je vais sur la grille du socle commun le palier 1, pas le palier 1, la compétence 1, donc votre maîtrise, votre compétence, qui est aussi un peu la nôtre, mais vous avez un niveau d'expertise sur certains items. Mais la globalité, oui, je regarde par exemple le savoir, quand on demande par exemple savoir décrire, évidemment le savoir décrire il y a une spécificité histoire-géographie, mais il y a une base de maîtrise de la langue.

On se situe, à ce stade de l'analyse, dans un cas de figure où le prescrit est connu (même partiellement) et pris en compte par l'enseignant de DNL.

\subsection{Au niveau du curriculum produit/planifié}

L'analyse des entretiens des 3 enseignants permet de mettre en évidence l'absence de planification des objectifs pédagogiques et des activités ou dispositifs d'apprentissage en lien avec la composante linguistique, et notamment la compétence PE. Par conséquent, elle ne donne aucune indication sur les SMK Langue et PK Langue mobilisées au niveau du curriculum produit.

Concernant les PCK Collaboratives, les éléments relevés dans les trois entretiens montrent, à propos de la prise en compte et du traitement de la composante linguistique, une absence de concertation, d'échanges, ou de démarches formalisées d'harmonisation (extrait 2) pour, par exemple, choisir et formuler collectivement les objectifs pédagogiques à atteindre, formuler, expliciter ou clarifier les contenus à enseigner, que ce soit entre enseignants d'une même discipline, entre enseignants de DNL différentes, ou que ce soit avec des enseignants spécialisés dans l'apprentissage de la langue, enseignants de français, enseignants spécialistes du Français Langue Etrangère (FLE) ou Langue Seconde (FLS) par exemple.

Extrait 2 (entretien E2) :

Q2.21 (...) Est-ce qu'il vous arrive parfois d'être en situation de partage, comme ça, de mise en commun?

E2.21 Non. Alors pourquoi ? Le pourquoi... Il y a des raisons vaseuses, et il y a des raisons personnelles. Pas intimes, mais personnelles.

\section{Q2.22 Liées à la conception des choses ? A la conception de la discipline?}

E2.22 Non. Liées à l'individu que je suis. Je suis un individu non pas égoïste, mais je suis un individualiste. (...) En revanche, je n'ai jamais hésité, jusqu'à aujourd'hui d'ailleurs, à échanger. Je suis une personne qui fonctionne beaucoup dans l'informel. Donc avec ma collègue, nous échangeons relativement fréquemment, mais c'est très informel.

Q2.23 C'était surtout ça qui m'intéressait, c'était surtout de savoir si c'était lié à des conceptions différentes sur le plan de la discipline, que vous enseignez (...).

E2.23 Non. Même si... Bon, ça n'est pas lié à ça. Elle a une pratique en revanche, elle écrit au tableau, elle écrit beaucoup son cours. Je ne le pratique pas. Forcément nous avons des 
pratiques qui diffèrent. N'empêche que les objectifs restent toujours les mêmes. On a un impératif. Mais c'est vrai qu'on devrait plus se... Plus collaborer (...).

E2.24 (...) On a un vrai cloisonnement, et c'est lié aux personnalités, aux personnes. Moi j'en fais partie, donc je reconnais clairement que... Réfractaire peut-être... Pas vraiment réfractaire. [...] Je suis dans l'informel. Qu'est-ce que je n'aime pas les formalités. Mais je suis plus pour les praticités. La praticité, le concret, direct. Nous sommes des personnes intelligentes, nous sommes des professionnels, nous connaissons notre métier. On a un besoin, on va se comprendre. Deux ou trois mots, deux ou trois échanges, on va finir par se comprendre. Et s'il y a un vrai besoin...

A ce stade de l'analyse, on se situe dans un cas de figure où le prescrit, pour ce qui concerne la composante linguistique, est connu et pris en compte par l'enseignant de DNL, mais non planifié au niveau du curriculum produit.

\subsection{Au niveau du curriculum réel}

L'analyse des entretiens ne permet pas de mettre en évidence clairement au niveau du curriculum réel les indicateurs d'une mobilisation de PCK Langue (SMK et PK). Nous rappelons que les indicateurs des SMK Langue se situent au niveau des notions et des concepts utilisés par l'enseignant. Les indicateurs de PK Langue se situent au niveau des objectifs pédagogiques définis et présentés aux élèves, des consignes, des types d'activités, des guidages, des modalités de mise en œuvre, et de l'évaluation. Sur ce point, une observation in situ en situation d'enseignement-apprentissage des pratiques effectives des enseignants aurait sans doute permis une analyse plus fine et aurait pu permettre la mise en évidence de pratiques inconscientes et involontaires ou non prévues, intégrant la composante linguistique. Toutefois, ce constat ne signifie pas pour autant que la dimension linguistique n'est pas prise en compte par les enseignants de DNL dans le cadre de leur enseignement et de leurs pratiques pédagogiques. Il montre plutôt une variabilité dans la prise en compte et l'intégration de la composante Langue, et la mise en place de stratégies individuelles ponctuelles (extrait 3) pour remédier à des besoins constatés (par exemple choisir de dicter ou non la trace écrite pour favoriser le passage de l'oral à l'écrit en insistant sur la ponctuation et sur les désinences et marqueurs grammaticaux, revenir sur un point de grammaire, de conjugaison, une règle d'orthographe), plus que sur l'application de choix pédagogiques et didactiques définis collectivement et fondés sur une connaissance des concepts, des contenus, des approches méthodologiques et des possibilités de mise en œuvre pédagogique (guidage, supports pédagogiques, stratégies d'évaluation...).

\section{Extrait 3 (entretien E2) :}

E.2.6 (...) Je suis une discipline non linguistique, on va dire ça.

\section{Q2.7 Considérée comme...}

E2.7 Considérée... Et bien je préfère le mot « considérée », parce que finalement dans mes pratiques je fais beaucoup de français, enfin le français entre guillemets, pas de littérature, quoique de temps en temps j'en fais un tout petit peu avec eux...

\section{Q.2.8 La langue?}

E.2.8 Mais la langue... Mes cours, étant donné qu'ils sont dictés et non pas notés au tableau, je prends un énorme plaisir à rouler les $\mathrm{R}$, à allonger les $\mathrm{L}$, quand il y a $2 \mathrm{~L}$, à allonger les $\mathrm{M}$ quand il y a $2 \mathrm{M}$, les $\mathrm{N}$. Je pense dans mon verbiage, mon verbage, l'orthographe, qu'ils sentent bien qu'il y a une orthographe, et je les habitue à tous les niveaux de la $6^{\text {ème }}$ à la $4^{\text {ème }}$, parfois 
avec les $3^{\text {ème }}$. Avec les $3^{\text {ème }}$, j'ai pas trop le temps, mais de la $6^{\text {ème }}$ à la $4^{\text {ème }}$, je ponctue mes phrases, je les ponctue toujours par... «Elles ont été interpellées. Interpellées ? . Et là j'entends "ées », parce que dès le début de l'année je les ai habitués ainsi. Maintenant dans mon discours, dans mon cours, dans la trace écrite qu'ils prennent, je ponctue ça de leçons grammaticales, de leçons orthographiques, de leçons de conjugaison, les terminaisons, voilà.

Sur ce point, les informations relevées au cours de l'analyse des entretiens tendent à montrer que cette variabilité dans la prise en compte et le traitement de la composante linguistique est à mettre en corrélation avec la sensibilité, le profil et le parcours personnels de chaque enseignant (extrait 4), et n'est donc pas conditionnée par une démarche d'expertise et de rationalisation des pratiques associée à la maîtrise de PCK Langue.

\section{Extrait 4 (entretien E2) :}

Q2.17 Et une question, par rapport à ça, c'est-à-dire à la conscience que tu as des besoins du public, de leurs difficultés éventuelles, et donc des points spécifiques à cibler concernant la langue, est-ce que tu peux m'expliquer comment ça t'es venu, est-ce que tu as été formé à ça, est-ce que tu as suivi des formations pour ça ? Est-ce que tu t'es auto formé ? Voilà, j’aimerais que tu puisses m'évoquer ça un peu.

E2.17 C'est très empirique, et je pense que c'est lié aussi à la personnalité. C'est très lié à ma personnalité. Mon histoire, mon vécu jouent beaucoup dans mon approche professionnelle. Nous sommes tous les deux de l'ancien système scolaire, de l'ancien système d'apprentissage scolaire, où la place de la langue était primordiale, peut-être que tu n'as pas connu ça? Personnellement, j'ai été formé à la langue française, j'ai été éduqué à la langue française. Je me rends compte, maintenant que j'ai 35 ans, et quand j'ai débuté j'en avais 23 ans, que tout naturellement, c'était une évidence, le respect des règles grammaticales, lexicales, syntaxiques, XXX. C'était une évidence, les élèves ne l'avaient pas, quand j'étais confronté à eux, quand je continue à être confronté à eux encore plus maintenant.

Ce constat peut être également relié à un certain nombre de difficultés identifiables, qui ne permettent pas à l'enseignant de DNL de mobiliser les PCK nécessaires à une prise en compte adaptée et efficiente de la dimension linguistique, et plus spécifiquement, pour ce qui concerne notre étude, des compétences PE. Ces difficultés sont essentiellement dues à une méconnaissance des SMK Langue et un déficit dans la maîtrise des PK Langue et relèvent du degré d'expertise des enseignants de DNL dans le domaine de l'apprentissage de la langue (E2.11). Elles sont clairement identifiées pour ce qui concerne par exemple l'évaluation des productions des apprenants (E1.23, extrait 5).

\section{Extrait 5 (entretien E1) :}

E1.22 Surtout la rédaction. Je trouve que les enfants ont énormément de mal à rédiger des phrases, sujet, verbe, complément, quelque chose qui est simple, et facile à comprendre. Ils font souvent très compliqué.

\section{Q1.23 Tu l'évalues ça ?}

E1.23 L'année dernière j'avais commencé, avec la mise en place du livret de compétences. J'ai lâché l'affaire, parce que c'était, comment dire, je commençais à leur enlever 2, 3 points sur chaque copie. Et encore, je comptais large, j'enlevais 1 demi-point par moins 10 fautes. J'ai laissé tomber. Mais en même temps je reconnais que ça me pose un vrai problème, parce que leurs copies sont bourrées de fautes, et que quelque part c'est comme si on leur ouvrait la trappe. Du moment que je comprends et que j'ai les deux mots de vocabulaire qu'on attend, je 
te donne le point. Mais c'est pas un cadeau que je leur fais non plus. Mais en même temps ça me semblait injuste de leur enlever trop de points.

Elles expliquent également, par exemple, les difficultés pour l'enseignant de DNL à définir des objectifs pédagogiques et des attentes précises en termes de productions à réaliser, d'où une tendance à fixer des exigences en inadéquation avec les besoins des apprenants au niveau des compétences PE (production de phrases simples, focalisation sur la compétence lexicale plus que sur la compétence méthodologique/pragmatique mobilisée en situation de production, ou fréquence peu importante des situations de productions de textes).

A ce stade de l'analyse, on se trouve dans un cas de figure où le prescrit, pour ce qui concerne la composante linguistique, est connu et pris en compte par l'enseignant de DNL, non planifié au niveau du curriculum produit, et intégré au curriculum réel, mais de manière inadaptée ou insuffisante au regard des besoins des apprenants. Les indicateurs auxquels nous nous intéressons ci-dessous, qui concernent les PCK Expertise, apportent quelques éclairages sur la capacités des enseignants à identifier et analyser les difficultés auxquelles ils sont confrontés, liées à la fois au profil des apprenants, et aux compétences nécessaires.

\subsection{Les PCK Expertise}

L'analyse des trois entretiens permet de mettre en évidence deux types d'indicateurs de PCK Expertise mobilisées. Les premiers concernent la capacité des enseignants à identifier et à cibler les types de difficultés et les besoins des apprenants dans le domaine de la maîtrise de la langue à l'écrit. Dans les trois entretiens, ces difficultés sont mises en lien avec l'origine socioculturelle des apprenants et avec le contexte sociolinguistique (extrait 6), même si les problématiques liées au contact français-créole ne sont pas explicitées et analysées de manière détaillée.

\section{Extrait 6 (entretien E1) :}

E1.44 (...) C'est difficile, des fois des mots tout simples ils vont mal les prononcer, alors que ce sont des mots qu'ils connaissent, parce qu'ils ne les ont jamais vus écrits. Donc après forcément, quand tu fais de l'histoire... La géographie c'est plus ça. Mais l'histoire, (...) y a forcément un vocabulaire que tu n'utilises pas tous les jours Donc oui, c'est difficile. Et pour moi c'est en grande partie culturel.

\section{Q1.45 Socioculturel, lié à l'origine sociale, au contexte social, familial ?}

E1.45 De toute façon la richesse du vocabulaire que tu acquiers, ça dépend pour beaucoup de la famille, comment on te parle, quels mots on emploie, est-ce qu'on parle français déjà, ou est-ce qu'à la maison c'est en créole. Je crois que tout ça ça joue.

Les autres indicateurs montrent la capacité des enseignants à identifier les difficultés auxquelles eux-mêmes sont confrontés dans la prise en compte de la dimension linguistique dans le cadre de leur spécialité. Dans les trois entretiens, les enseignants reconnaissent l'utilité, voire la nécessité d'intégrer la composante Langue et de contribuer davantage au développement et à l'acquisition des compétences linguistiques, à l'écrit notamment, des apprenants dans leur spécialité respective. Ils reconnaissent également le besoin pour y parvenir d'une meilleure expertise dans ce domaine, notamment par le biais d'une formation adaptée (E3.32), mais également par le biais d'une collaboration plus étroite et formalisée entre enseignants de DNL et avec des enseignants experts (E2.23), ou encore par la mise en application d'une approche 
décloisonnée des apprentissages (extrait 7) qui tendrait vers une démarche intégrative, ou la conception d'un élargissement de spécialité des enseignants de DNL.

\section{Extrait 7 (entretien E3) :}

E3.32 Moi je pense qu'il faudrait carrément décentraliser les matières. Là pour le moment c'est... Le problème c'est que je ne suis pas assez compétent pour faire du français, et tu le seras pas pour faire de la SVT. Mais il est là le problème. Parce que ça, ce que je fais, tu pourrais le faire. Le travail sur les textes tout ça. Mais derrière il faut avoir les connaissances. Sinon tu te retrouves bloqué. Alors est-ce qu'il faudrait leur ajouter du français en $6^{\text {ème }}, 5^{\text {ème }} \ldots$

Les indicateurs des PCK Expertise mobilisées par les enseignants sont en cohérence avec l'analyse des SMK et PK mobilisées au niveau du curriculum réel, à savoir une (re)connaissance des besoins des apprenants au plan linguistique, des difficultés identifiées pour les enseignants de DNL à répondre à ces besoins dans les pratiques réelles déclarées, liées à une méconnaissance des SMK Langue et un déficit dans la maîtrise des PK Langue, et enfin une capacité à cibler la nature de ces difficultés et à proposer des pistes de réflexion pour améliorer la situation existante et répondre aux besoins des apprenants.

\section{Discussion}

L'analyse des PCK mobilisées par les enseignants des DNL histoire, géographie et SVT, pour ce qui relève de l'intégration de la composante linguistique au niveau PE permet de mettre en évidence plusieurs éléments. Elle montre tout d'abord une prise en compte seulement partielle et limitée de la dimension linguistique au niveau du curriculum prescrit. Elle semble ensuite montrer, au niveau du curriculum produit, l'absence de planification d'objectifs et de pratiques pédagogiques liées à la dimension linguistique. Elle montre enfin, au niveau du curriculum réel, l'intégration dans les pratiques pédagogiques déclarées d'une dimension linguistique, variable selon les enseignants, et globalement insuffisante au regard des besoins identifiés des apprenants. Cette analyse permet de mettre en évidence un déficit dans la maîtrise des SMK Langue et des PCK Langue. En d'autres termes une expertise insuffisante dans le domaine de l'apprentissage de la langue aux niveaux didactique et pédagogique des enseignants de DNL pour leur permettre de mobiliser les compétences nécessaires pour intégrer efficacement et de manière adaptée la composante linguistique au niveau PE dans l'enseignement de leur spécialité respective. Les indicateurs relatifs aux PCK Expertise relevés dans les discours des enseignants montrent d'ailleurs leur capacité non seulement à identifier la nature de ces lacunes, mais également à exprimer des besoins potentiels pour améliorer l'existant, notamment en termes de formation, de partage et de transfert de compétences dans le cadre de démarches collaboratives, et en termes de reconfiguration disciplinaire par le décloisonnement et la transversalité.

L'analyse des PCK enseignantes mobilisées semble donc mettre en évidence les difficultés, pour les enseignants de DNL à transposer les contenus prescrits dans leurs pratiques effectives, et à intégrer la dimension linguistique dans leur spécialité respective aux différents niveaux curriculaires, en fonction des besoins des apprenants. Les indicateurs relevés montrent l'absence d'un curriculum produit intégrant la dimension linguistique, caractérisé par la non planification des objectifs et des pratiques potentiels en lien avec la composante Langue. Toutefois, ce constat ne peut être considéré indépendamment des données issues de l'analyse du curriculum prescrit, qui devra constituer un volet complémentaire à notre recherche. Nous formulons en effet l'hypothèse qu'un déficit d'information et de précision au niveau des contenus programmatiques de chaque discipline pourrait constituer un obstacle à la prise en 
compte de la dimension linguistique dans l'enseignement des DNL et à la transposition du curriculum prescrit au réel.

L'une des réponses possibles dans le contexte d'enseignement-apprentissage considéré dans le cadre de notre étude, consisterait donc dans la réorganisation de la construction curriculaire des DNL. Par réorganisation de la construction curriculaire, on entend l'implantation d'une phase curriculaire intermédiaire entre le curriculum prescrit et le curriculum réel : le curriculum produit (Martinand, 2003). Celui-ci est envisagé comme un curriculum local, opérationnel et transversal, intégrant la dimension linguistique de manière rationalisée en fonction des besoins des apprenants, produit par l'ensemble des enseignants de DNL en collaboration avec des enseignants experts dans le domaine de la langue, dans une démarche de partage et de transfert de compétences.

Cette réflexion amène à repenser les contenus et les enjeux des DNL en termes de choix et de finalités curriculaires. Elle conduit à envisager l'intégration de la dimension linguistique dans les DNL comme un élargissement de spécialité, impliquant une extension et une réorganisation des contenus à enseigner (Coquidé, 2011). Dans cette perspective, la sensibilisation et la formation des enseignants de DNL à ce domaine, à ses problématiques et à ses enjeux, apparaît comme une condition sine qua non pour permettre une évolution vers une prise en compte adaptée et efficiente de la langue dans le cadre des DNL, et par là même favoriser la construction et le développement des compétences en français des apprenants dans le cadre scolaire. On tendrait alors vers une approche caractérisée par une problématique d'intégration Langue-DNL visant à exploiter les potentialités que présentent les DNL comme un espace d'exposition à la langue et de pratique de la langue dans une perspective acquisitionnelle (Noyau, 2007) et dans une démarche d'économie cognitive, pédagogique et didactique (Cavalli, 2008 ; Wokusch, 2008).

\section{Conclusion}

Dans l'état actuel, les résultats de cette étude de cas demeurent provisoires et ne permettent pas de tirer de conclusions définitives. La recherche que nous avons menée relève d'une démarche exploratoire et expérimentale. Elle doit être envisagée non pas comme un produit fini et comme une fin en soi, mais au contraire comme le point de départ potentiel d'une réflexion qui devra faire l'objet d'un approfondissement et d'un élargissement à plusieurs niveaux. Si nous avons privilégié au niveau méthodologique l'analyse du discours et des pratiques planifiées et réelles déclarées des enseignants, nous considérons cependant que l'analyse du curriculum produit réel élaboré par les enseignants (descriptifs de séances et de séquences pédagogiques, cahiers de bords, fiches d'activités élèves, etc.) et des pratiques réelles des enseignants observées in situ en situation d'enseignement-apprentissage permettrait de compléter et d'affiner les données que nous avons recueillies tant au niveau quantitatif que qualitatif. Par ailleurs, notre recherche porte sur un échantillon constitué de trois enseignants de DNL dans un établissement cible. Il serait intéressant d'appliquer notre cadre de recherche à un échantillon plus large et plus important, qui permettrait d'envisager une approche comparative et la prise en compte d'autres variables et d'autres critères d'analyse en lien par exemple avec le type d'établissement, le niveau et le type de public ciblé, le profil des enseignants ou encore la structure des équipes pédagogiques. Ajoutons que ce cadre d'analyse n'est pas figé et qu'il doit être envisagé non pas comme un modèle fermé à usage unique, mais au contraire comme un modèle transférable et applicable à d'autres contextes d'enseignement-apprentissage, d'autres champs disciplinaires et d'autres spécialités. 
Si les résultats de notre étude restent à compléter et à approfondir, ils constituent néanmoins une source potentielle d'informations qui peut permettre dans l'état actuel d'envisager des pistes de réflexion et d'exploitations au niveau local, sur certains aspects de la problématique liée à l'appropriation du français en contexte scolaire par le biais des DNL et à la complémentarité DL-DNL. Dans la continuité de cette étude, la mise en place de projets expérimentaux de type interdisciplinaire/transdisciplinaire axés sur les principes de décloisonnement et de chevauchement des disciplines, serait une perspective intéressante. Elle permettrait à la fois de vérifier la pertinence des données que nous avons présentées et d'observer in situ les processus à l'œuvre dans une approche de ce type.

\section{Références bibliographiques}

Anciaux, F., Caliari, P., Alin, C., Le Her, M. et Fery, Y.-A. (2005). Imagerie visuelle et rappel moteur : Effet du bilinguisme français/créole. Psychologie française, 50(4), 419-436.

Anciaux, F. (2007). Langues de présentation des consignes et performances motrices chez des bilingues français/créole aux Antilles françaises. Revue Canadienne des Sciences $d u$ Comportement, 39(4), 315-325.

Anciaux, F. (2008). Alternance des langues et stratégie d'enseignement en EPS en contexte bilingue. Recherches et Ressources en Éducation et en Formation, 2, 25-33.

Anciaux, F. (2008). Alternance codique français/créole en EPS dans la Caraïbe. Dans T. Karsenti, R-P. Gary et A. Benziane (dir.), Former les enseignants du XXIe siècle dans toute la Francophonie (p. 29-41). Clermont-Ferrand: Presses Universitaires Blaise Pascal.

Anciaux, F. (2010). Vers une didactique de l'alternance codique aux Antilles françaises. Actes du Colloque International Spécificités et diversité des interactions didactiques : disciplines, finalités, contextes 2010. Lyon : INRP.

Bardin, L. (1996). L'analyse de contenu. Paris : Presses Universitaires de France.

Blanchet, P., Moore, D. et Asselah Rahal, S. (2008). Perspectives pour une didactique des langues contextualisée. Paris: Éditions des Archives Contemporaines et Agence Universitaire de la Francophonie.

Brohy, C. (2008). Didactique intégrée des langues : évolution et définitions. Babylonia, 1, 9-11.

Cambrone, S. (2004). Contact de langues en milieu scolaire. L'alternance codique en situation de classe: quelles stratégies? IRD/AREC-F, Université des Antilles-Guyane (Fort-deFrance, Martinique). Consulté le 20/01/2013 sur: http://www.mq.ird.fr/pdf/AREC-FCambrone.pdf.

Candelier, M. (2008). Approches plurielles, didactiques du plurilinguisme : le même et l'autre. Les cahiers de l'ACEDLE, 5(1), 65-90.

Causa, M. (2007, juin). Enseignement bilingue. L'indispensable alternance codique. Le français dans le monde, 351, 18-19.

Causa, M. (2009). Enseignement d'une LE et d'une DNL : mettre en place une compétence discursive dans la production écrite de niveau avancé. Synergies Roumanie, 4, 179-188.

Cavalli, M. (2005). Education bilingue et plurilinguisme. Le cas du Val d'Aoste. Paris : Didier.

Chiss, J.-L. (2010). La didactique des langues : contacts et méthodologies. Synergies, 1, 93-96.

Coquidé, M. (2011). Etude sur l'élargissement de la spécialité enseignante dans l'enseignement intégré de science et de technologie (EIST) au collège. Lyon: Institut Français de l'Éducation.

Direction Générale de l'Enseignement Scolaire (2011). Grille de référence des compétences et des connaissances, palier 3. Paris : Ministère de l'Éducation Nationale.

Duverger, J. (2005). L'enseignement en classe bilingue. Paris : Hachette.

Gajo, L. (2001). Immersion, bilinguisme et interaction en classe. Paris : Didier. 
Gajo, L. (2008). L'intercompréhension entre didactique intégrée et enseignement bilingue. Dans Conti, V. et Grin, F. (dir.), S'entendre entre langues voisines: vers l'intercompréhension (p. 131-150). Genève : Georg.

Inspection Générale de l'Éducation Nationale (2002). Les modalités de scolarisation des élèves non francophones nouvellement arrivés. Rapport $\mathrm{n}^{\circ} 2002-029$. Paris: Ministère de l'Education Nationale.

Inspection Générale de l'Éducation Nationale (2007). Les livrets de compétences : nouveaux outils pour l'évaluation (rapport n²007-048). Paris : Ministère de l'Education Nationale.

Martinand, J.-L. (2003). L'éducation technologique à l'école moyenne en France : problèmes de didactique curriculaire. La revue canadienne de l'enseignement des sciences, des mathématiques et des technologies, 3(1), 100-116.

Ministère de l'Éducation Nationale. BO spécial $N^{\circ} 10$ du 25 avril 2002.

Noyau, C. (2007). L'enseignement de disciplines non linguistiques en FLS au primaire en Afrique de l'ouest : les sciences d'observation à travers les manuels et les activités de classe. Tréma, 28, 49-61.

Prudent, L.-F., Tupin, F. et Wharton, S. (2005). Du plurilinguisme à l'école. Vers une gestion coordonnée des langues en contextes éducatifs sensibles. Berne : Peter Lang

Sabourin, P. (2003). L'analyse de contenu. Dans B. Gauthier (dir.), La recherche sociale : de la problématique à la collecte des données. Sainte-Foy : Presses de l'Université du Québec.

Sainton-Fachtum, J. (2008a). Réflexion pour une didactique adaptée de l'enseignement du français. Dans Maiga, A. (dir.), Le français dans les aires créolophones. Vers une didactique adaptée. Paris : L'Harmattan

Sainton-Fachtum, J. (2008b). Les contraintes phonologiques en lecture en milieu de diglossie créole-français. Recherches et Ressources en Education et en Formation, 2, 35-46.

Shulman, L.-S. (1986). Those who understand : Knowledge Growth in Teaching. Educational Researcher, 15(2), 4-14.

Verdelhan-Bourgade, M. (2002). Le français de scolarisation. Pour une didactique réaliste. Paris : Presses Universitaires de France.

Vigner, G. (1989). Le Français, langue de scolarisation. Diagonales, 12. 41-45.

Vigner, G. (2001). Enseigner le français comme langue seconde. Paris : CLE International.

Wokusch, S. (2005). Didactique intégrée : vers une définition. Babylonia, 4, 14-16.

Wokusch, S. (2008). Didactique intégrée des langues (étrangères) à l'école : vers l'enseignement des langues de demain. Prismes, Revue pédagogique de la HEP, 8, 30-34. 